\title{
Cated Box, una tecnología para hacer estudios de movilidad 2.0
}

\author{
Abel Rionda ${ }^{1}$, David Martínez ${ }^{1}$, Xabiel G. Pañeda ${ }^{2}$, David Arbesú ${ }^{1}$, J. Emilio Jimenez \\ F. F. Linera ${ }^{2}$ \\ \{abel.rionda, david.martinez, david.arbesu\}@adnmobilesolutions.com, \{xabiel, \\ linera\}@uniovi.es, emilio.jimenez@fivelines.es \\ ${ }^{1}$ ADN Mobile Solutions, Parque Científico Tecnológico, 33203, Gijón/Xixón, Asturies, España. \\ ${ }_{2}^{2}$ Universidad de Oviedo, Campus de Gijón, 33204, Gijón/Xixón, Asturies, España. \\ 3 Fivelines, Parque Científico Tecnológico, 33203, Gijón/Xixón, Asturies, España.
}

DOI: $10.4304 /$ risti.10.97-110

Resumen: en los últimos años el aumento de desplazamientos en vehículo privado ha generado importantes problemas de congestión tanto en ciudades como en vías interurbanas. Asimismo, estos desplazamientos han producido importantes problemas de estacionamiento. Para ser capaces de realizar predicciones y definir políticas que puedan mejorar la circulación, las administraciones realizan cada cierto tiempo estudios de movilidad que analicen la situación del tráfico y propongan medidas correctoras. En este artículo se presenta una tecnología que permite la realización de estudios de movilidad a partir de la colaboración del conductor-ciudadano como elemento clave para obtener el conocimiento. La facilidad de uso de la tecnología, la motivación y la recompensa son elementos que deben de tenerse muy presentes si se desea mantener en el conductor el interés a colaborar. La tecnología Cated Box se ha probado con éxito en el estudio de movilidad del área tecnológica de la ciudad de Gijón conocida como Milla del Conocimiento.

Palabras clave: e-gobierno; estudio de movilidad; vehículos; teléfono móvil; Internet de las cosas.
Abstract: in the last few years an important increase in private car journeys has created significant congestion in cities and on interurban roads. Furthermore, these shifts have generated significant parking problems. To be able to make predictions and define policies that can improve circulation, governments from time to time perform mobility studies that analyze the traffic situation and propose corrective measures. In this paper we present a technology that enables studies of mobility using the driver-citizen collaboration as the key element to obtain knowledge. The ease of use of technology, motivation and reward are elements that must be thoroughly considered if you want to keep in the driver's interest to cooperate. Cated Box technology has been tested successfully in the study of 
mobility in technological area of the city of Gijón known as "Milla del Conocimiento".

Key-words: e-government; mobility study; vehicle; cellular phone; Internet of things.

\section{Introducción}

Los problemas de congestión, aparcamiento y contaminación generados por los vehículos de combustión, han llevado a las administraciones públicas a hacer estudios que permitan mejorar la integración de los turismos en el entorno urbano e interurbano. Mediante el análisis de los hábitos en los desplazamientos se pretende, por un lado dotar de infraestructuras a las ciudades y regiones para ser capaces de absorber dichos flujos de vehículos, y por otro, proponer medidas alternativas que puedan en cierta medida mitigar el uso del vehículo particular.

Estos estudios se han venido realizando a través de diferentes combinaciones de medios como por ejemplo, los sistemas de conteo, bien con cables o con cámaras, o mediante encuestas a los conductores. Sin embargo, esta forma de llevar a cabo los estudios de movilidad presenta importantes inconvenientes debido a su coste. En el caso de los sistemas de conteo, su principal limitación radica en que hacen un análisis puntual, es decir, estudian el tráfico en la vía en la que se sitúa el sistema. Si se quieren controlar varias, es necesario hacer el despliegue en cada una de ellas. Para acotar los costes, los sistemas de monitorización suelen limitarse a las zonas principales de paso, quedando el resto fuera del estudio.

En este artículo se presenta una tecnología que permite desarrollar los estudios de movilidad de una forma innovadora. A través de la colaboración del conductor y con un coste relativamente bajo, es posible monitorizar los movimientos de los vehículos a cualquier hora y en cualquier zona. Esta colaboración se buscará incorporando en el sistema de monitorización del vehículo una herramienta para mejorar la conducción que permita ahorrar en combustible. De esta forma las administraciones públicas y el ciudadano llegarán a un modelo de colaboración "win-win".

El sistema que aquí se presenta, denominado Cated Box, se ha probado en un estudio de movilidad para una zona de la ciudad de Gijón (Asturies/España) denominada "Milla del Conocimiento" en la que se sitúa la Universidad y una parte muy importante de las empresas tecnológicas de la zona. El estudio se desarrolló durante mes y medio arrojando unos resultados muy interesantes, tanto desde el punto de vista del estudio de movilidad, como desde el de los conductores participantes, que consiguieron un ahorro de combustible de aproximadamente del 10\%.

El resto del artículo se organiza de la siguiente forma. La sección 2 recoge los trabajos relacionados en el campo de los estudios de movilidad y los sistemas de monitorización de vehículos. La sección 3 presenta la tecnología Cated Box tanto desde el punto tecnológico como de la estimulación del conductor a la participación. La sección 4 describe el estudio de movilidad realizado como prueba de concepto de la tecnología y la sección 5 incluye las conclusiones y los trabajos futuros. 


\section{Trabajos relacionados}

El desarrollo de estudios de movilidad es una herramienta que administraciones públicas y organizaciones han venido utilizando desde hace tiempo con diversos objetivos como, el análisis de la congestión, aparcamiento, uso de los diferentes medios de transporte, horarios, seguridad, etc.

Clásicamente, las fuentes de información de estos estudios han sido la monitorización de vías mediante cámaras (Archetti, 2006) (Schrank, 2011) (Klakhaeng, 2011) o sensores en el asfalto (Kent, 2010), encuestas (York, 2006) (Novák, 2007) o simplemente mediante modelos (Singh, 2006) dependiendo el objetivo del estudio de movilidad.

Solo algunos sistemas orientados al análisis de congestión han atacado el problema con una metodología diferente. Por ejemplo, algunos sistemas han introducido el uso del GPS, bien a través de dispositivos específicos (Schrank, 2011) (Usman, 2010), o bien a través de aplicaciones para el móvil (Varandas, 2010). Algunos estudios experimentales han utilizado sistemas de monitorización a través la triangulación con las antenas de telefonía móvil (Calabrese, 2011). Sin embargo, la mayoría de los estudios de movilidad siguen utilizando métodos de conteo y monitorización tradicionales por la complejidad de motivar al usuario a colaborar. Solo los sistemas de gestión de flotas, donde el conductor está obligado a colaborar, utilizan sistemas de monitorización en el propio vehículo (Thong, 2007) (Kargupta, 2010).

Este punto débil, que ha evitado la evolución metodológica de los estudios de movilidad, es uno de los principales objetivos de la tecnología que aquí se presenta. Cated Box busca que el conductor saque provecho del uso del sistema mediante la reducción del consumo de combustible, y de esta forma estimular su participación en este tipo de iniciativas mediante una filosofía "win-win" administración/ciudadano. La idea subyacente será la misma que la de iniciativa denominada waze (www.waze.com) que mediante un entorno colaborativo intenta aportar información a los conductores sobre el estado de las carreteras. Sin embargo, mientras que Cated Box busca que cada conductor trabaje en su propio beneficio (reducción del consumo), en el caso waze el conductor genera beneficio a la comunidad (proporcionando rutas), y es ella, la que le corresponde (con rutas del resto de conductores) generando un beneficio indirecto. Esto puede hacer que el interés en colaborar no se mantenga de forma permanente, produciendo problemas similares a los de las redes P2P (Herrera, 2007)(Binzenh, 2007). Por ello, en Cated Box se ha buscado que el beneficio que pueda obtener el conductor sea directo y no tenga dependencias con el resto de usuarios.

\section{La tecnología Cated Box}

La tecnología Cated Box ha sido desarrollada para tener al usuario conductor como elemento central de todo el proceso de recogida de datos del estudio de movilidad. Puesto que un conductor debe de estar motivado para ofrecerse a colaborar gratuitamente, el sistema además tiene la vertiente de ofrecer al conductor el realizar una conducción más eficiente, con el consiguiente ahorro en combustible. De esta forma, los beneficios serán tanto para la administración como para el conductor. 
La arquitectura general de la tecnología Cated Box, como se muestra en la figura 1, está compuesta por diversos componentes tanto hardware como software:

- Sistema abordo (on-board):

- Sensor Cated para la recogida de datos del vehículo.

- Sistema de recepción, recomendación y transmisión del teléfono móvil.

- Sistema central:
- Sistema de almacenamiento y análisis off-line de datos.
- Portal Web de asistencia al usuario.
- Cuadro de mando y panel de control para la administración pública.

El sistema abordo está compuesto de dos elementos principales. El sensor Cated que se conecta a la centralita del coche y permite leer datos sobre el funcionamiento del vehículo y el asistente a la conducción que se encarga de recibir la información del GPS del teléfono y del sensor Cated, realizar diversos tipos de análisis, asistir al conductor y enviar la información al sistema central.

El sensor Cated se comunica con la centralita del turismo a través del estándar OBD-II obligatorio en todos los vehículos europeos desde 2001 (2003 para diesel). (SAE, 1998). El dispositivo es capaz de extraer diversas variables funcionamiento del vehículo, como velocidad o revoluciones por minuto, y transmitirlas al teléfono móvil a través de una conexión Bluetooth. Dicha información es transmitida aproximadamente cada 2 segundos. El sensor se alimenta desde el propio interfaz OBD-II, lo que permite mantenerlo siempre conectado al vehículo y olvidarse del mismo. Además no necesita ningún tipo de configuración más allá de la interconexión inicial con el teléfono móvil (localización y pareado del Bluetooth).

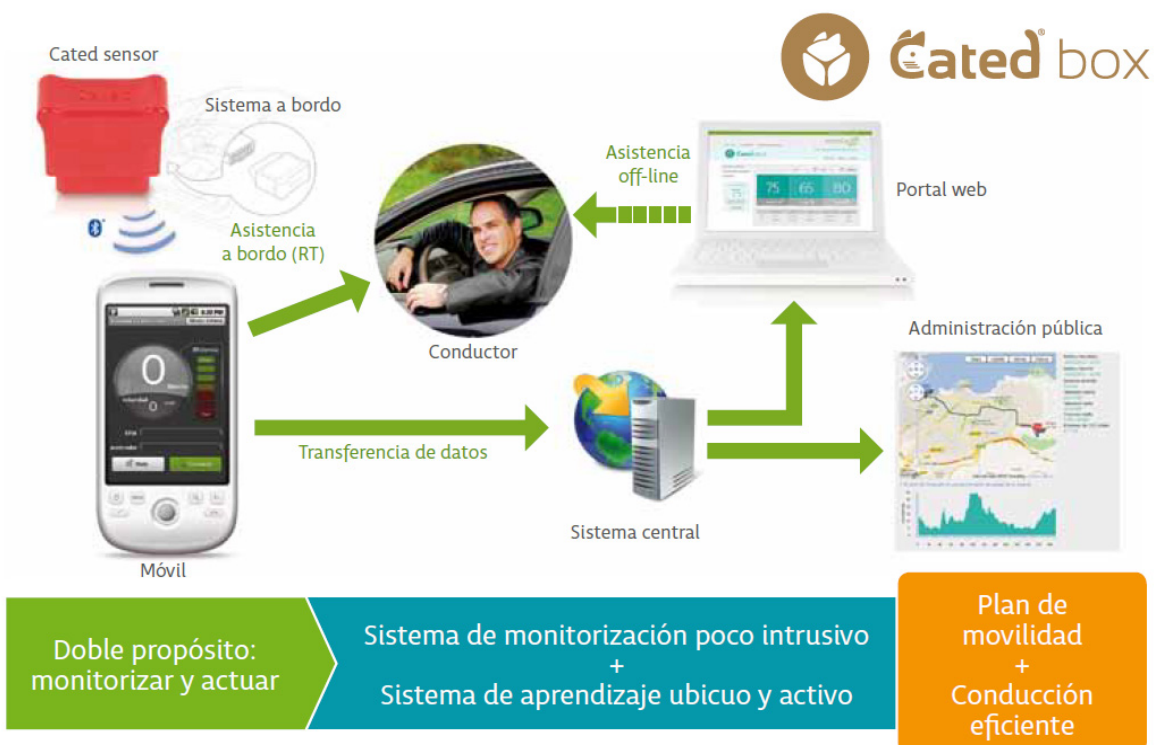

Figura 1 - Arquitectura general del sistema Cated Box Sistema abordo 
El asistente a la conducción es un programa con diversas responsabilidades:

- Asistir al conductor durante sus trayectos en coche indicándole la marcha más eficiente desde el punto de vista de ahorro de combustible. Está pensado para que el teléfono móvil en el que se ejecuta se coloque en un soporte similar al utilizado para los navegadores al lado del volante de modo que no haga al conductor apartar la vista de la carretera. El sistema incorpora dos tipos de avisos, los visuales y los sonoros o acústicos. Los visuales presentan en un círculo que varía de color el número de la marcha recomendada, como se muestra en la figura 2. El círculo de color que puede ser verde, naranja o rojo indicará si el conductor sigue la recomendación o se aleja de ella.

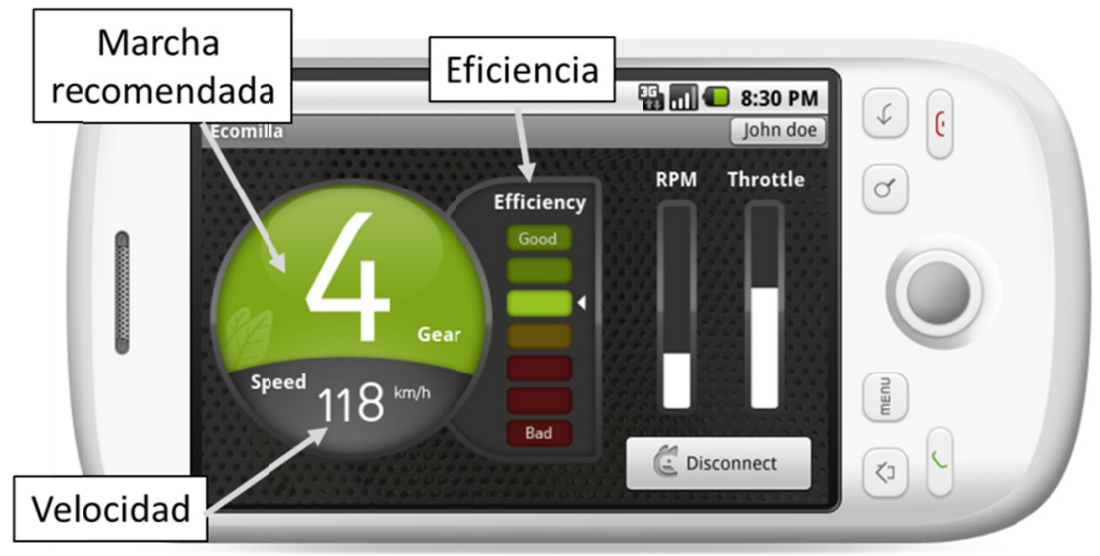

Figur: 2 - Sistema abordo

- Recoger información procedente del sensor GPS del teléfono móvil y de la centralita del coche para enviarlo al sistema central donde pueda ser procesado y utilizado con posterioridad tanto el propio conductor del vehículo como por parte de las administraciones públicas (de forma impersonalizada).

Para poner en marcha el asistente, el conductor simplemente tendrá que seleccionar la aplicación en el teléfono y pulsar el botón conectar. Una vez realizado el proceso de conexión el sistema no requerirá ninguna otra interacción con el mismo.

En cuanto a las características técnicas, la aplicación móvil está desarrollada en el sistema operativo móvil Android y contiene una serie de algoritmos que necesitan las siguientes entradas y producen las siguientes salidas:

\section{Entradas}

- Base de datos de vehículos con datos técnicos de modelos (Relación de marchas, potencia, cilindrada, peso, coeficiente aerodinámico, etc.). Esta información se descarga del servidor cuando se configura el perfil de usuario (con su vehículo asociado).

- Lectura de datos de la centralita del vehículo a través del sensor OBD-II y mediante el protocolo Bluetooth (Velocidad, RPM, carga del motor, presión del turbo, 
acelerador, MAF/Mass Air Flow, etc.). Información de posicionamiento GPS capturada a través del sensor del propio teléfono móvil.

\section{Salidas}

- Cálculo del consumo de combustible, instantáneo y medio, inducido a partir de los datos de entrada.

- Indicación en tiempo real, de la marcha recomendada, aplicando técnicas de conducción eficiente.

- $\quad$ Registro en local y posterior envío de toda la información recogida, tanto entradas como salidas, a un servidor para su posterior análisis detallado y extracción de conclusiones globales y particulares.

\subsection{Sistema central: almacenamiento y análisis off-line de datos}

El sistema central es el encargado de recibir la información de los dispositivos móviles de los conductores que utilizan el servicio de conducción eficiente. La aplicación instalada en el teléfono móvil se conectará a un servicio activo en el sistema central y le transferirá los datos recogidos desde la última conexión. Dichos datos serán registrados en una base de datos de tipo Apache CouchDB que presenta unos parámetros de escalabilidad muy adecuados para sistemas que manipulan grandes cantidades de información. Esta base de datos almacenará la información accesible a los usuarios. El sistema central se completará con una base de datos SQLServer donde se estructurará un DataWareHousing sobre el cual se realizarán los procesos de análisis orientados a la administración que requieren agregaciones y cálculos complejos. De esta forma ell sistema orientado a los usuarios no se verá penalizado por los procesamientos que puedan realizarse para proveer de información a las administraciones públicas.

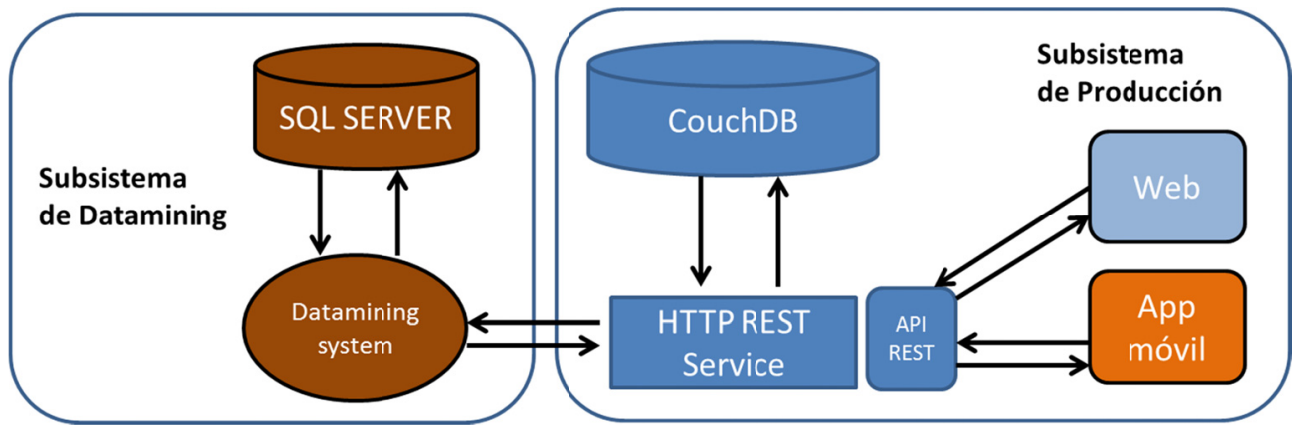

Figura 3 - Arquitectura del Sistema central Cated Box

En la figura 3 se presenta la arquitectura física del sistema central de Cated Box. El sistema está estructurado para soportar una alta escalabilidad. Por un lado el subsistema de producción, encargado de registrar la actividad continua del sistema y proporcionar acceso a los usuarios, está compuesto por una base de datos CouchDB y un servidor Web al que se le ha dotado de una API REST a través de la cual las aplicaciones móviles y Web acceden al sistema. El sistema de datamining, que se encarga de realizar los análisis complejos de la actividad de los conductores, está diseñado utilizando SQL Server Analysis Services y la base de datos SQL Server. El 
sistema se despliega sobre dos máquinas siendo la primera Linux Debian, para el sistema de producción, y la segunda Windows Server que soporta el sistema de minerỉa de datos.

\subsection{Portal Web de asistencia al usuario}

El portal Web es el componente del sistema que permite al conductor analizar su conducción, tanto desde el punto de vista de la movilidad cómo desde el punto de vista de la conducción eficiente.

Para ello, el portal se estructura en diversas zonas, una de ellas para el análisis de los itinerarios, en el que podrá observar sus rutas y valorarlas (figura 4). Esta información será proporcionada de forma anónima a la administración para que pueda utilizarla en sus estudios de movilidad. En otra de las secciones, el usuario puede analizar la eficiencia de su conducción tanto de forma general, como pormenorizada por trayecto. De esta forma podrá ver diversos índices de conducción eficiente (figura 4), $\mathrm{CO}_{2}$ emitido a la atmósfera o el coste económico de sus desplazamientos.

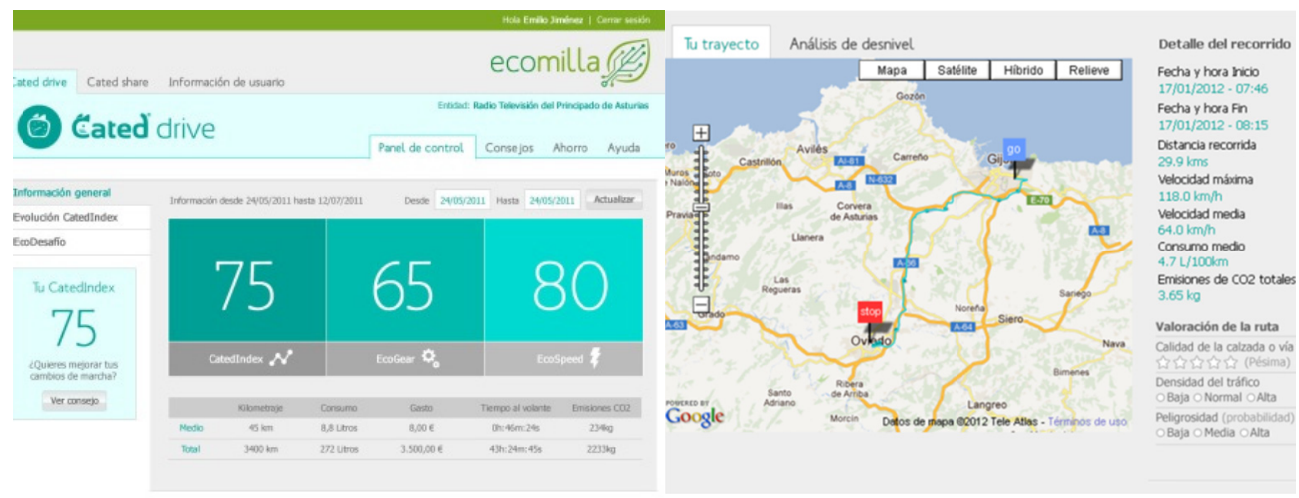

Figura 4 - Portal Web de asistencia al usuario

El conductor también dispondrá de una sección para recibir consejos de mejoras en su conducción y una zona de atención al usuario, donde podrá presentar quejas, realizar consultas o dar de alta incidencias en el funcionamiento del servicio.

\subsection{Portal Web para la Administración Pública}

El portal para la administración pública está dividido en dos entornos independientes. Por un lado el sistema de gestión, que permite dar de alta los usuarios y realizar las tareas de atención los mismos (modificaciones, quejas, sugerencias, incidencias, actividad o inactividad, etc.); por otro, el cuadro de mando, que permiten visualizar los indicadores tanto para el estudio de movilidad en tiempo real, como para el análisis de eficiencia en la conducción.

Los indicadores relativos a la movilidad son los siguientes:

- Frecuencia sobre intervalo de tiempos de itinerario. 
- Itinerarios por día totales.

- Kilómetros por día totales.

- Número de trayectos por usuario.

- Trayectos urbanos e interurbanos por día.

- Orígenes desde barrios o ciudades (configurable).

- Horas de llegada y salida a zonas (configurable).

Estos indicadores podrán ser discriminados por: edades, género, sectores de actividad laboral, etc.

En cuanto a los indicadores de eficiencia la información recogida es la siguiente:

- Consumo medio por día.

- CatedIndex (métrica de eficiencia) medio por día.

- CatedIndex urbanos e interurbanos por día.

- EcoGear y Eco Speed (métricas de eficiencia) medio por día.

- Inventario dinámico de emisiones de $\mathrm{CO}_{2}$ y litros de combustible ahorrados por todos los vehículos gracias a las recomendaciones de conducción eficiente frente a la situación de partida (diagnóstico inicial).

Para cada uno de los indicadores, el cuadro de mando incluye representación gráfica y tabular (pudiendo ser exportada esta información a formato Excel para facilitar posible tratamiento posterior). También es posible el filtrado de datos por rangos de fechas. En la figura 5 se muestran algunas pantallas del cuadro de mando.
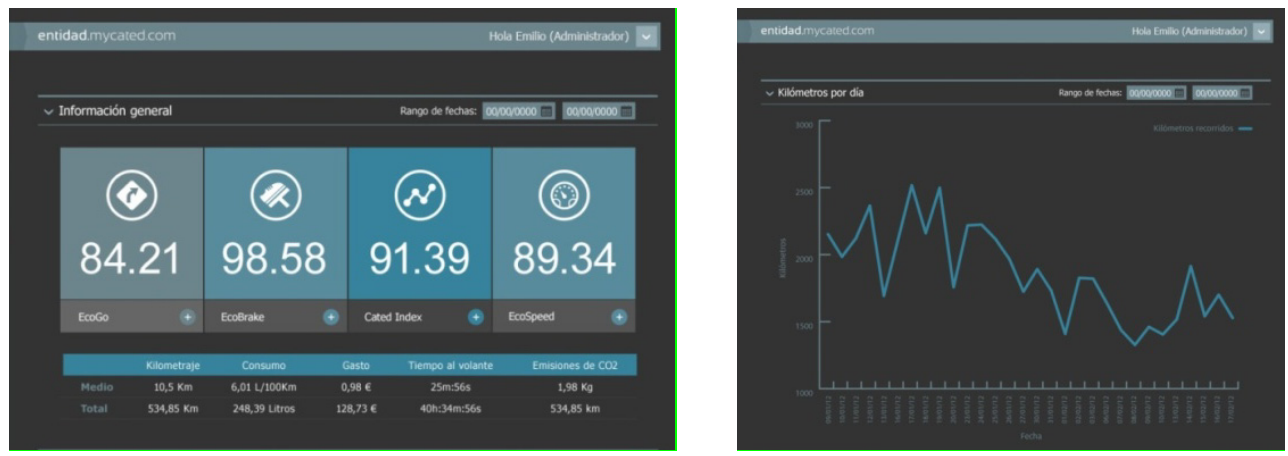

Figura 5 - Interfaz cuadro de mando para la administración

Puesto que el acceso a información sobre la movilidad de los conductores podría invadir la intimidad de los mismos, la aplicación garantiza que los datos proporcionados están anonimizados y agregados, de forma que no es posible estudiar el patrón de comportamiento de un conductor de forma individual.

\section{Caso de Estudio: Movilidad en la "Milla del Conocimiento"}

La evaluación del sistema Cated Box se ha desarrollado en el contexto del proyecto Ecomilla (www.ecomilla.com). En dicho proyecto se monitorizaron y asistieron a 150 conductores procedentes de las instituciones (Universidad de Oviedo, UNED, RTPA, Parque Científico Tecnológico de Gijón, Hospital de Cabueñes, Jardín Botánico 
Atlántico, Laboral Ciudad de la Cultura) que conforman la Milla del Conocimiento en la Ciudad de Gijón (figura 6). Dicho experimento se realizó con dos enfoques, por un lado realizar un estudio de movilidad para mejorar los sistemas de comunicación de dicha zona, y por otro, buscar la mejora de la conducción de los trabajadores de la zona (Rionda, 2012), lo cual debería ser el incentivo de los conductores a participar (modelo "win-win"). El seguimiento y análisis de los datos se realizó únicamente en días laborables, puesto que el estudio buscaba analizar la movilidad en una zona de clara actividad laboral.

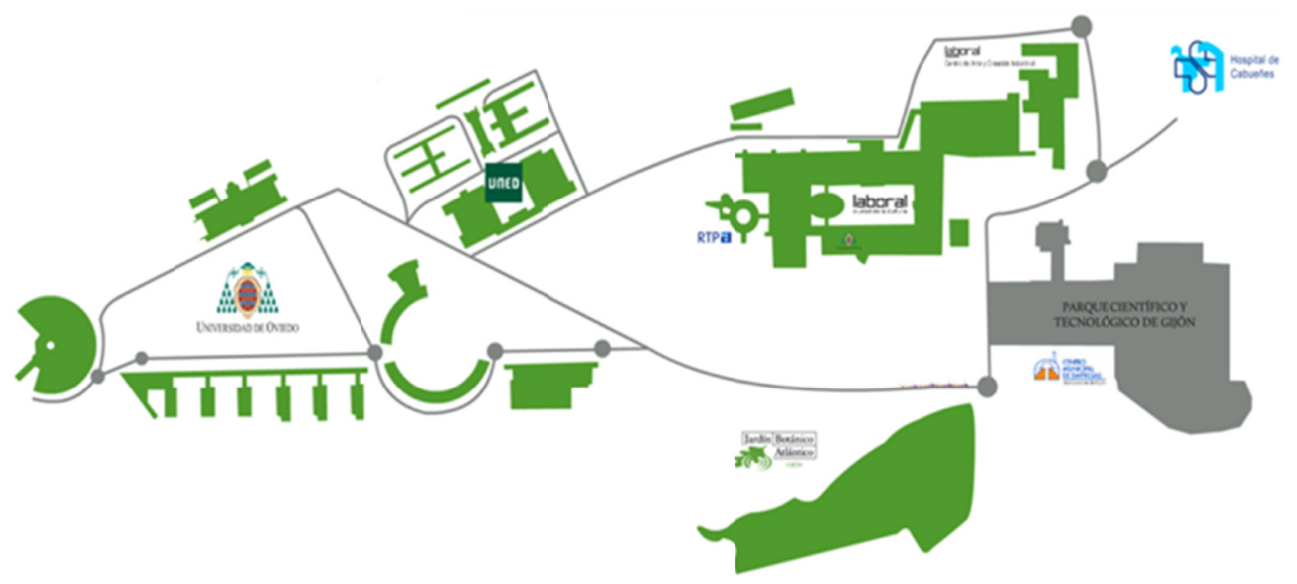

Figura 6 - Mi lla del Conocimiento (Gijón)

Los conductores pertenecientes a centros de trabajo de la Milla del Conocimiento se presentaron voluntarios para participar en el estudio y aportaron diferentes datos personales para poder realizar un análisis estratificado de los datos recogidos. El estudio se realizó durante 30 días durante los cuales se recogió un número de entre 110 y 220 trayectos diarios.

En lo que se refiere a la conducción eficiente, el estudio se dividió en dos fases, una en la que no se ofrecieron recomendaciones a los conductores (modo caja negra, 5 días de duración) para ser tomada como línea de referencia y otra en la que el sistema (modo asistente) ofreció las recomendaciones para mejorar su forma de conducir.

\subsection{Resultados}

El estudio de movilidad sobre los trabajadores de la Milla del Conocimiento ha permitido un detallado estudio de su comportamiento a la hora de utilizar el coche. Se han podido determinar horarios de salida, de entrada, carreteras de acceso, zonas de salida y llegada de los trabajadores, calles más transitadas en los trayectos urbanos y las vías interurbanas principales, etc. Algunos de los mismos se relatan a continuación:

- Se pudo constatar que los itinerarios en general son bastante cortos, teniendo en cuenta que la mayoría de estos tienen una duración menor a 15 minutos, como se observa en la figura 7. Dada la configuración urbana de la zona central de Asturias, este dato es una indicación clara de que los trayectos urbanos o 
semiurbanos son los predominantes. El 58\% de los de los trayectos se realizan dentro de la ciudad de Gijón, siendo los más numerosos los tránsitos desde el barrio de El Llano y la zona rural, con un 9\% cada uno. En cuanto al resto de Asturias, se pudo constatar que un $13 \%$ de los trabajadores y estudiantes de la "Milla" provienen de la zona centro (Oviedo, Siero, Llanera, Avilés y Villaviciosa) y sólo un 4\% de las cuencas mineras (Mieres, Langreo, Aller, etc.).

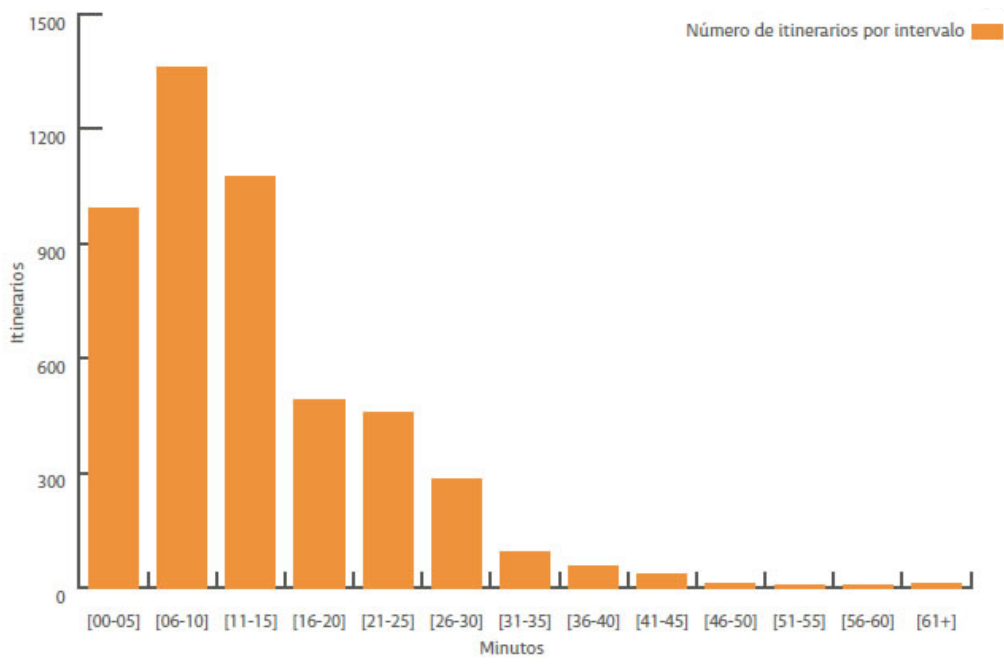

Figura 7 - Duración de los trayectos analizados

- Otro elemento analizado fue el nivel de ocupación de las distintas vías de acceso a la Milla del Conocimiento. Los resultados revelaron que el punto más utilizado para llegar a la zona es la carretera N632 en dirección al Jardín Botánico (figura 8), utilizada en un 38\% del total de los trayectos. A ésta le siguen, ambos con un 25\% de uso, los accesos desde la Autovía del Cantábrico (A-8) por Deva y Viesques, es decir, las salidas 382 y 385 respectivamente. Por último, la vía de entrada a la "Milla" menos utilizada es la Avenida de la Pecuaria, con un $10 \%$ de trayectos sobre la cifra global. El $2 \%$ residual se correspondería con vías de menor relevancia, como la Carretera de Santurio o el Camino del Piqueru.

- Los horarios de entrada y salida de los trabajadores y estudiantes de la "Milla" revelaron que, en el periodo de lunes jueves, existen dos franjas horarias de máxima densidad de tráfico muy bien definidas. De esta manera, se aprecia que entre las 07:00 y las 09:59 de la mañana se producen un 46\% del total de los trayectos diarios con destino la Milla del Conocimiento. Entre las 14:00 y las 16:59 se dan otro $21 \%$ de los desplazamientos de entrada, lo que lleva a la conclusión de que un porcentaje importante de trabajadores y estudiantes comen fuera del área de la "Milla", bien en sus casas o bien en restaurantes cercanos.

- Respecto a las salidas, siguiendo en la línea del patrón comentado anteriormente, se observó que entre las 13:00 y las 16:59 se producen un 33\% del total, explicado tanto por el abandono de la Milla durante las horas de 
comida como por el hecho de que ciertas entidades pueden tener jornada continua durante toda la semana. Por otra parte, en la franja horaria comprendida entre las 17:00 y las 20:59 se registra un significativo $44 \%$ de salidas, teniendo un peso claramente mayor el intervalo entre las 17:00 y las 19:59.
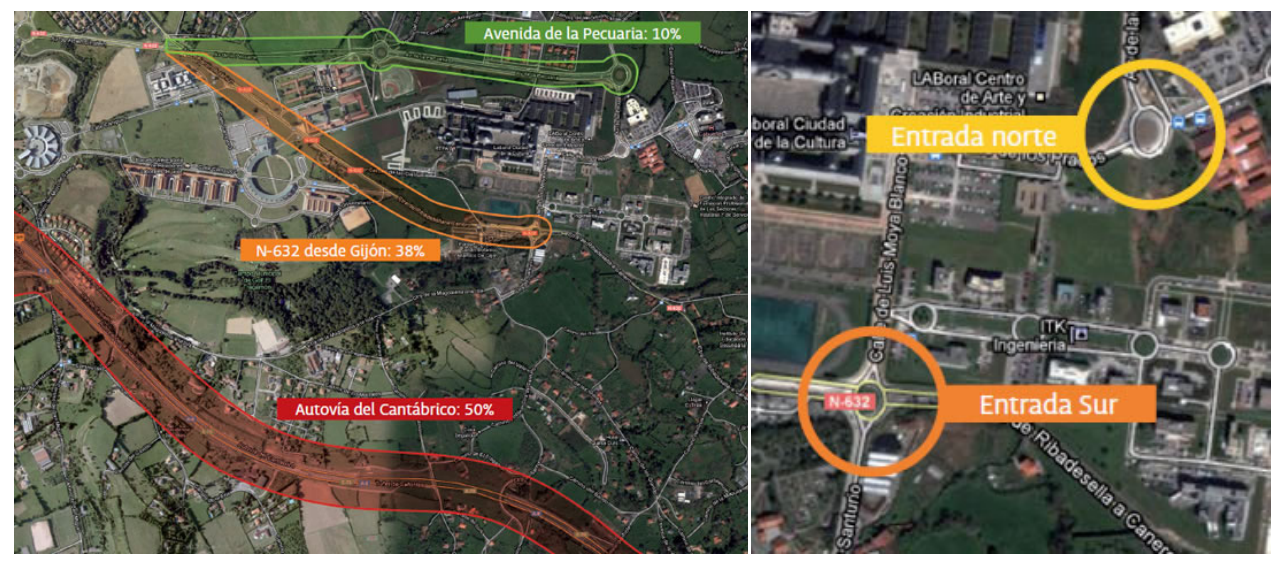

Figura 8 - Vías de acceso a la Milla del Conocimiento y entradas al Parque CientíficoTecnológico de Gijón

- En lo que se refiere específicamente al Parque Científico-Tecnológico (Zona empresarial de la "Milla"), se ha observado que en todas las franjas horarias la carga de tráfico soportada por la entrada Sur es evidentemente superior a la que se refleja en la entrada Norte (figura 8).

En lo que se refiere a los resultados relacionados con la conducción eficiente, se analizaron diferentes métricas de eficiencia y se cruzaron con los datos asociados a horarios, trayectos, género, etc. En términos generales el dato observado más destacable fue una reducción media de combustible del 10\%. En la figura 9 se observa la evolución del consumo medio durante los periodos de caja negra (primeros 5 días) y de asistencia. 


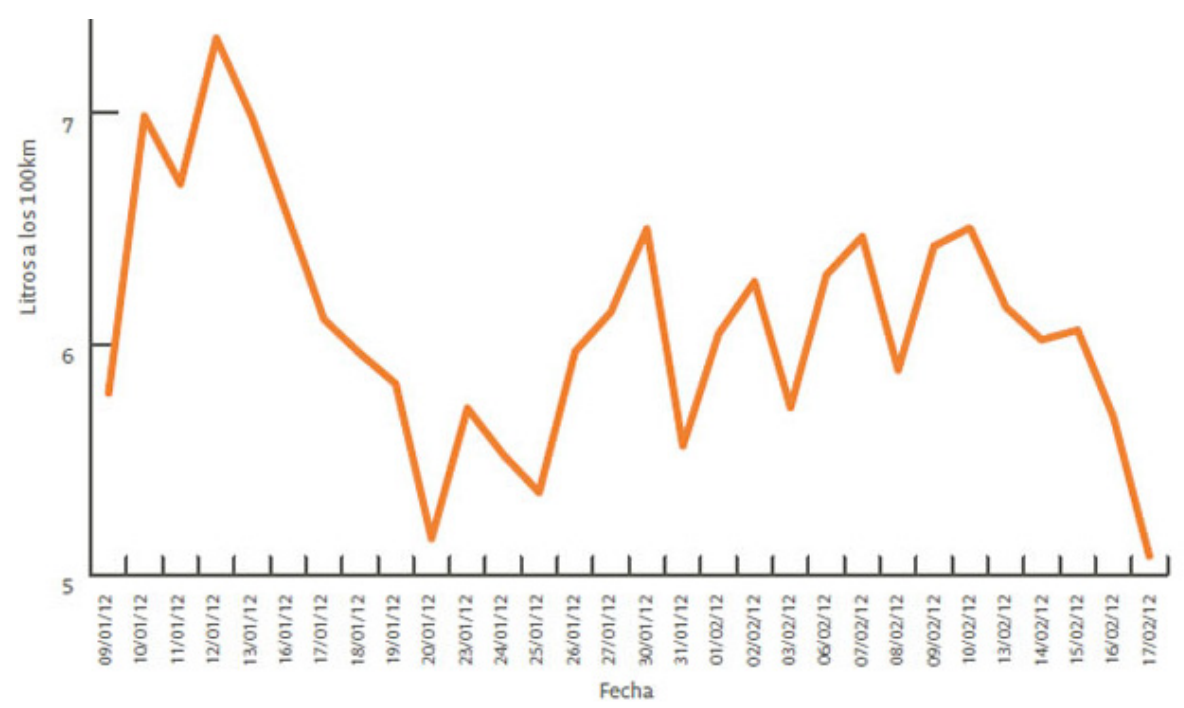

Figura 9 - Consumo medio diario

Además del análisis de los datos objetivos recogidos con el Cated Box, el estudio ha sido utilizado para preguntar a los conductores por la usabilidad de la tecnología y su experiencia como participantes del proyecto "Ecomilla". Para evaluar sus impresiones se les realizó una encuesta voluntaria cuyas preguntas fueron las siguientes:

1. ¿Con qué nivel siguió las recomendaciones del asistente?

2. ¿Considera que ha mejorado su conducción?

3. El estudio nos arrojó una media de ahorro en combustible del $10 \%$, ¿Usted sintió reflejado este ahorro en su bolsillo?

4. ¿Cómo calificaría el uso del sensor y la aplicación móvil?

5. ¿Cuál es la valoración global que le da al proyecto ecomilla?

6. ¿Participarías en un proyecto similar extendido a toda la ciudad?

7. Sabemos que la motivación es importante en la participación. ¿Cómo podemos motivar la participación constante?

De los 150 conductores 46 respondieron la encuesta, siendo los resultados más importantes los siguientes:

- La mayoría de los conductores consideraron que el sistema era fácil o muy fácil de usar. 5 conductores señalaron que su manejo era sencillo una vez se acostumbraron y dos que el uso era complejo.

- La pregunta de si volverían a participar en un estudio similar, dos usuarios respondieron que no, 5 que tal vez y el resto que sí.

- En cuanto al ahorro de combustible 7 conductores indicaron que no habían ahorrado y el resto se repartieron entre menos y más del 10\%. 
- En lo que se refiere a la valoración global del proyecto (1-5), 15 lo valoraron con un 5, 22 con un 4, 4 con un 3, 2 con un 2 y 3 con un 1 .

\section{Conclusiones y trabajos futuros}

Como conclusiones podemos decir que el sistema Cated Box permite la realización de estudios de movilidad proporcionado información muy detallada y con un esfuerzo económico inferior al que supone el desarrollo de los mismos con sistemas más clásicos. Con una selección adecuada de los usuarios a monitorizar, es posible realizar análisis similares a los estudios de audiencia que se utilizan en la televisión. Además la forma de llevar a cabo el estudio permite el análisis de la movilidad de forma continua y la obtención de resultados prácticamente en tiempo real. La motivación de los conductores mediante algún tipo de recompensa, como por ejemplo la reducción en el consumo de combustible, es el factor clave que hace que la administración y ciudadano creen un entorno de colaboración "win-win".

A pesar de que los beneficios de la tecnología Cated Box han quedado demostrados en el estudio "Ecomilla", es necesario seguir trabajando en algunos aspectos. El primero de ellos, se centra en la adaptación de la tecnología para estudios de larga duración. Aspectos como aumentar la facilidad de uso, diseñar nuevos sistemas de análisis offline o la carga de datos en tiempo real mediante redes $3 \mathrm{G}$, pueden ser aspectos muy importantes para mantener la motivación a usuarios en periodos superiores a un mes.

Otro campo de trabajo futuro, será la adaptación del sistema para su uso en autobuses y resto de tráfico pesado. En este caso, puesto que dichos vehículos no cuentan con puerto de conexión OBD-II, será necesario adaptar el interfaz de comunicaciones.

\section{Referencias bibliográficas}

Archetti, F., Messina, E., Toscani, D., Vanneschi, L. (2006). Classifying and Counting Vehicles Traffic Control Applications. EvoWorkshops 2006, LNCS 3907, pp. 495499.

Calabrese, F., Colonna, M., Lovisolo, P., Parata, D., Ratti, C. (2011). Real-Time Urban Monitoring Using Cell Phones: A Case Study in Rome. Transaction on Intelligent Transportation Systems. IEEE.

Binzenh, A., Leibnitz, K. (2007). Estimating Churn in Structured P2P Networks. ITC 2007, LNCS 4516, pp. 630-641. Springer.

Herrera, O., Znati, T. (2007). Modeling Churn in P2P Networks. Proceedings of the 4oth Annual Simulation Symposium (ANSS'O7).

Kargupta, H., Sarkar, K., Gilligan, M. (2010). MineFleet ${ }^{\circledR}$ : an overview of a widely adopted distributed vehicle performance data mining system. 16th International Conference on Knowledge Discovery and Data Mining (ACM SIGKDD). 
Klakhaeng, N., Yaothanee, J., Sinthupinyo, S., Pattara-Atikom, W. (2011) Traffic Predition Models for Bangkok Traffic Data. The $8^{\text {th }}$ Electrical Engineering/Electronics, Computer, Telecommunications and Information Technology (ECTI).

Kent, (2010). Kent Travel Report. Kent County Council.

Novák, J., Lud]k Sỳkora L. (2007). A city in motion: time-space activity and mobility patterns of suburban inhabitants and the structuration of the spatial organization of the Prague metropolitan area. Journal compilation. Swedish Society for Anthropology and Geography.

Thong, S., Han, C., Rahman, T. (2007). Intelligent Fleet Management System with Concurrent GPS \& GSM Real-Time Positioning Technology. 7th International Conference on ITS (ITST '07).

Rionda, A., Martínez, D. Pañeda, X.G., Arbesú, D., Jimenez, E., Linera, F.F. (2012). Sistema tutor para la conducción eficiente de vehículos de combustión. Revista Iberoamericana de Tecnologías del Aprendizaje (RITA). IEEE Education Society.

SAE, (1998). J1962. ODB-II. Vehicle E E System Diagnostic Standards Committee.

Schrank, D., Lomax T., Eisele, B. (2011). TTI's 2011 Urban Mobility Report. Texas Transportation Institute.

Singh, S.K., (2006). Future mobility in India: Implications for energy demand and CO2 emission. Transport Policy. Elsevier.

Usman, M., Lim S. (2010). Privacy Implications of Automated GPS Tracking and Profiling. Technology and Society Magazine. 1932-4529/10. IEEE.

Varandas, L., Vaidya, B., Rodrigues, J (2010). mTracker: A Mobile Trancking Application for Pervasive Environment. 24th International Conferencce on Advanced Information Networking and Applications Workshops. IEEE.

York, (2006). Local Transport Plan 2006-2011. York, City of York. 